\title{
Bloqueo del erector de la espina en cirugía torácica pediátrica
}

\author{
Erector spinae plane block in thoracic pediatric surgery \\ Sandra Lataste¹, Alvaro León'1, Carolina Campillay²
}

\begin{abstract}
Thoracotomy is associated with intense pain. In the pediatric population, the pain affects the ventilatory mechanics, which is also strongly influenced by the characteristics of the respiratory tract and chest according to the age. Therefore, regional techniques are strongly recommended. In 2016, ESP is described for the first time, which generates extensive sensory block in the chest wall, without approaching the pleura and the neuroaxial space from the technical point of view. At present, there is increasing experience in different surgical settings, but it is still scarce in pediatric patients. We present the successful application of the technique in 2 pediatric cases of thoracic surgery and various outcomes are described.
\end{abstract}

\section{RESUMEN}

La toracotomía está asociada con intenso dolor. En la población pediátrica, el dolor afecta la mecánica ventilatoria, que además se ve fuertemente influida por las características propias de las vías respiratorias y del tórax según la edad. Por lo anterior, las técnicas regionales están fuertemente recomendadas. En 2016 se describe por primera vez el ESP, que genera bloqueo sensitivo extenso en la pared torácica, sin aproximarse desde el punto de vista técnico a la pleura y al espacio neuroaxial. En la actualidad, existe experiencia en aumento en diversos settings quirúrgicos, pero es aún escasa en pacientes pediátricos. Presentamos la aplicación exitosa de la técnica en 2 casos pediátricos de cirugía torácica, así como la descripción de diferentes resultados posoperatorios.

\section{Key words:}

Erector spinae plane block, pediatric anesthesia, thoracotomy

\section{Palabras clave:}

Bloqueo erector de la espina, anestesia pediátrica, toracotomía

\footnotetext{
Médico Anestesiólogo, Clínica Indisa.

Médico Cirujano, Becada Anestesiología UNAB.
}

Fecha de ingreso: 10 de marzo de 2019

Fecha de aceptación: 19 de marzo de 2019

\section{ORCID}

https://orcid.org/0000-0003-4267-1948

Correspondencia:

Dra. Sanda Lataste Werth

E-mail: samalawe@gmail.com 


\section{Introducción}

a toracotomía está asociada con intenso dolor, atribuible a las incisiones del plano muscular, retracción o resección de las costillas y daño a los nervios intercostales. Si este estimulo nociceptivo se maneja de manera subóptima puede empeorar la disfunción pulmonar experimentada por la mayoría de estos pacientes[1].

En la población pediátrica esto es particularmente relevante ya que la mecánica ventilatoria se ve fuertemente influida por las características propias de las vías respiratorias y el tórax según la edad. En los lactantes la respiración es menos eficaz; el pequeño diámetro de las vías respiratorias aumenta la resistencia al flujo aéreo, la resistencia es inversamente proporcional al radio elevado a la cuarta potencia en un flujo laminar y a la quinta potencia para un flujo turbulento. La vía respiratoria del lactante es muy distensible y está mal sustentada por las estructuras circundantes. La pared torácica también es muy distensible, de manera que las costillas proporcionan escaso soporte a los pulmones; es decir, la presión negativa intratorácica se mantiene con dificultad y, por último, el espacio muerto es proporcionalmente similar a la de los adultos, pero el consumo de oxígeno es dos o tres veces mayor.

Por lo anterior, las técnicas regionales están fuertemente recomendadas[2] para reducir el uso de opiáceos y los efectos adversos relacionados, incluyendo hipoventilación, sedación, náuseas y vómitos[1].

La analgesia epidural torácica y el bloqueo paravertebral han sido la primera opción para el manejo del dolor postoracotomía, sin embargo, estas técnicas pueden ser desafiantes de realizar y están asociadas con una tasa significativa de fallos (sobre el $15 \%$ en el caso de la epidural en adultos)[3]. La anestesia epidural dorsal y la instalación de catéter para infusión continua no son técnicas que se utilicen habitualmente en niños, ya que las indicaciones se limitan a la cirugía torácica y abdominal alta, pero existe el riesgo de dañar la médula espinal[17].

Las opciones de rescate incluyen bloqueos intercostales y analgesia sistémica, ambas con sus limitaciones[3]. El bloqueo intercostal en niños no está recomendado cuando hay problemas de oxigenación o intercambio de gases[17], y es necesario mantener a todos los pacientes bajo observación debido al peligro del neumotórax tardío.

En 2016, la publicación de "The Erector Spinae Plane Block, A Novel Analgesic Technique in Thoracic Neuropathic Pain"[4] describe por primera vez una técnica exitosa en el manejo de dolor neuropático to- rácico. En ésta, el anestésico local es depositado en el plano profundo del músculo erector de la espina y superficial a la punta del proceso transverso. Alcanza un extenso bloqueo sensorial multi dermatoma por anterior, lateral y posterior de la pared torácica[5] sin aproximarse desde el punto de vista técnico a la pleura y al espacio neuroaxial[1],[3]. Su efecto analgésico parece aparecer debido a la difusión del anestésico local al espacio paravertebral, afectando tanto la rama ventral y dorsal de los nervios espinales torácicos[4], como también a las ramas comunicantes de la cadena simpática, lo que provee manejo del dolor visceral.

Posterior a ese año, hemos visto la emergencia de nuevas aplicaciones en diferentes "settings" quirúrgicos y de dolor, tanto agudo como crónico, del adulto. Entre ellos, fracturas costales[4], reparación de hernias abdominales[7], cirugía de mama[8],[9], cirugía de vertebras torácicas[10], cirugía bariátrica[15] y todo tipo de cirugías que involucren el tórax. Dentro de lo último se ha reportado su uso en lobectomías por videotorascoscopías (VATS)[11] y toracotomías abiertas por neumotórax persistente[12] o causas oncológicas[3].

Poco a poco, el interés se ha extendido a los casos pediátricos, como el manejo del dolor postoperatorio en cirugía oncológica torácica[5], pectum excavatum[16] y recientemente en plicatura diafragmática toracoscópica por un grupo chileno[13], en niños de 7, 6 y 3 años respectivamente.

Recientemente fue publicado en España el caso clínico de un lactante de 15 meses[14], en el que se utilizó la técnica asociada a infusión continua del anestésico local.

\section{Casos clínicos}

Presentamos el caso de dos pacientes pediátricos sometidos a cirugía torácica, donde se utilizó la técnica de bloqueo erector de la espina (ESP).

El primer caso es una lactante menor de 11 meses 11 días, nacida de término, con antecedentes de neumonías a repetición y SBOR en tratamiento con fluticasona 125 ug c/12 h. Ingresa para cirugía programada el 28/11/2017 con diagnóstico de malformación adenomatoidea quística asociada a secuestro pulmonar que involucra lóbulo inferior derecho y segmento basal posterior. Además, presenta una atelectasia parcial de lóbulo superior derecho. Equipo quirúrgico no solicita ventilación monopulmonar por lo que se intuba sin incidentes con tubo estándar, sin bloqueador bronquial. Mantenciónanestésica con gases y bolos de fentanilo. 
Se realiza toracotomía en sexto espacio intercostal derecho, posicionada en decúbito lateral izquierdo. Presenta sangrado intraoperatorio de aproximadamente 200 cc por lo que se transfunde con glóbulos rojos. Al finalizar la cirugía se instala tubo pleural N¹2 en el séptimo espacio intercostal derecho más trampa de agua a - $5 \mathrm{~cm} \mathrm{H}_{2} \mathrm{O}$.

En prono y aún con anestesia general, se realiza bloqueo erector de la espina bajo visión ecográfica con transductor lineal de 6-13 Mhz (SonoSite Edge; SonoSite, Inc., Bothell, WA, USA). Se identifica proceso transverso de $\mathrm{T} 2$ con transductor en posición sagital paramedial derecha y se punciona en plano con aguja stimuplex ${ }^{\circledR}$ D $50 \mathrm{~mm}$ con dirección de cefálico a caudal llegando y contactando con proceso transverso de T4. Tras aspiración se administran $3 \mathrm{cc}$ de levobupivacaína al 0,5\% (Chirocaína 5 mg/mL; Abbvie, Elverum, Norway). Posterior a ello, paciente fue extubada y transferida a la UCI pediátrica en buenas condiciones generales, tranquila y sin llanto, escala FLACC 0/10.

Se realiza primer control de dolor 1 hora posterior a ingreso a $\mathrm{UCl}$, paciente con FLACC 0/10. A las 12 horas paciente estable en lo hemodinámico y en lo ventilatorio, apoyada sólo con naricera a bajo flujo $(0,5 \mathrm{l} / \mathrm{min})$ y analgesia endovenosa con paracetamol a $15 \mathrm{mg} / \mathrm{kg} /$ dosis $\mathrm{c} / 6 \mathrm{~h}$ y ketorolaco 0,5 mg/kg/dosis c/8 h. Radiografía de tórax de control muestra parénquima pulmonar bien expandido a izquierda a derecha con espacio residual por lobulectomía y resto del parénquima bien expandido (Figura 1). Se mantiene con FLACC 0/10. Se realimenta oral sin problemas a las 4 horas postoperatorio. A las $24 \mathrm{~h}$ se mantiene estable, sin requerimientos de oxígeno, con dolor controlado con analgesia endovenosa, sin opioides, FLACC 2/10.

A las $48 \mathrm{~h}$ post cirugía se retira drenaje pleural y se da de alta de manera satisfactoria al tercer día post operatorio.

El segundo caso corresponde a un escolar de 5 años, con antecedentes de convulsión febril compleja y estudio negativo para epilepsia. Ingresa día 14/04/2018 por cuadro de 6 días de evolución caracterizado por fiebre persistente de $>39^{\circ} \mathrm{C}$, diagnosticándose una neumonía basal derecha más derrame pleural leve ipsilateral. Se hospitaliza, cursa con evolución tórpida a pleuroneumonía cavitada derecha con derrame pleural de alto grado, más insuficiencia respiratoria. El día 17/04/2018 ingresa a pabellón para videotoracoscopía derecha. Se monitoriza de manera estándar, se induce de forma inhalatoria, mantención con gases y fentanilo. Intubación monobronquial izquierda con fibrobroncoscopio, ventilación monopulmonar bien tolerada, Se posiciona en decúbito lateral

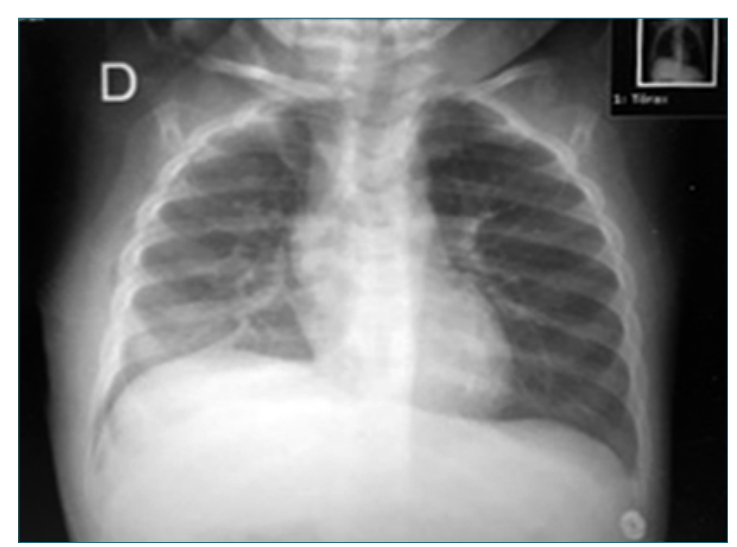

Figura 1. Reexpansión torácica completa y precoz post quirúrgica.

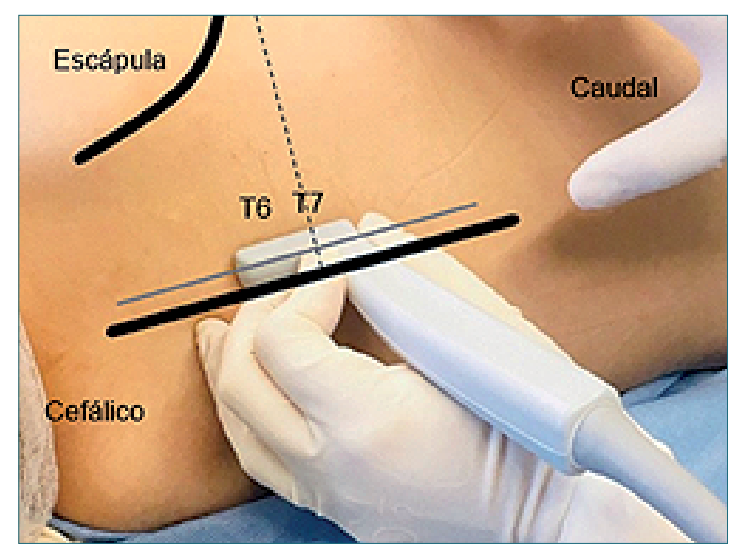

Figura 2. Búsqueda de posición, transductor en posición paramedical derecha en T6. Decúbito lateral derecho.

izquierdo. Incisión en $5^{\text {to }}$ Ell, introducción de trócar 5 $\mathrm{mm}$ dando salida a pus franca, posterior a ello introducción de cámara 5 mm 30 grados y neumotórax 5 $\mathrm{mmHg}$ bien tolerado; segundo port de $5 \mathrm{~mm}$ en $6^{\text {to }}$ Ell línea axilar posterior. Se le realiza drenaje, decorticación, aseo quirúrgico y se deja con drenaje pleural $16 \mathrm{Fr}$ aspirativo a $-10 \mathrm{~cm} \mathrm{H}_{2} \mathrm{O}$ en línea axilar posterior. En la misma posición, y aún con anestesia, se realiza bloqueo erector de la espina con transductor lineal de 6-13 Mhz (SonoSite Edge; SonoSite, Inc., Bothell, WA, USA), se identifica proceso transverso de T4 con transductor en posición sagital paramedial derecha (Figura 2 ), se punciona en plano con aguja stimuplex ${ }^{\circledR} \mathrm{D} 50$ $\mathrm{mm}$ con dirección de cefálico a caudal llegando y contactando con proceso transverso de T6. Tras aspiración se administran 6 cc de levobupivacaína al 0,25\% repartidos en T6, T7 Y T8 (Figuras 3 y 4). 


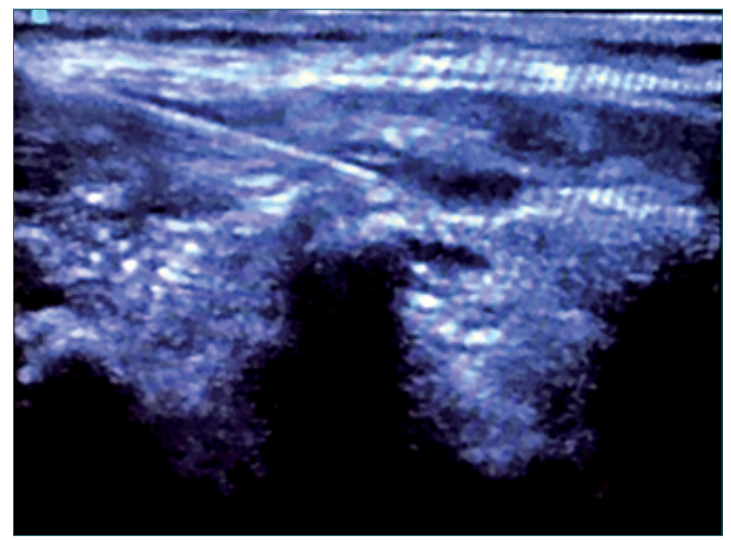

Figura 3. Aguja posicionada en relación con proceso transverso T7.

El paciente fue extubado en pabellón y transferido a UCI pediátrica en buen estado general, tranquilo y apoyado con mascarilla Venturi a $\mathrm{FIO}_{2} 35 \%$. Se apoya bloqueo regional con analgesia endovenosa en bomba de infusión continua de ketorolaco más metamizol y paracetamol ev. Control a las $12 \mathrm{~h}$, evoluciona estable, saturando $100 \%$ con naricera a 0,5 $\mathrm{L} / \mathrm{min}$, hemodinamia sin alteraciones, con dolor leve escala de caritas 3/10. Radiografía con pulmón derecho bien expandido, tolerando bien kinesioterapia,

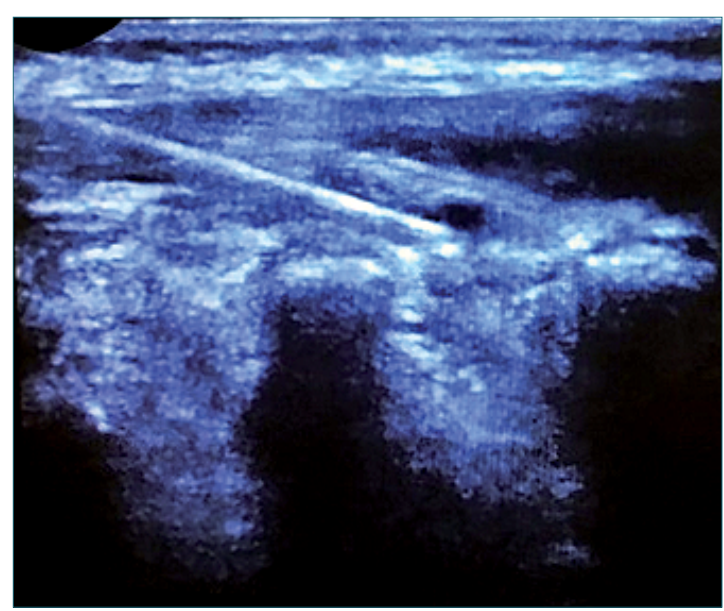

Figura 4. Inyección de anestésico local en relación con proceso transverso T7 y profundización a T8.

sin necesidad de analgesia de rescate. Se realimenta por vía oral sin problemas desde las 6 horas postoperatorias. Control de $24 \mathrm{~h}$, estable, sin dolor, se decide suspender BIC de analgesia quedando solo con paracetamol $15 \mathrm{mg} / \mathrm{kg} /$ dosis cada $6 \mathrm{~h}$. Drenaje pleural se retira el día 25/04/2018. Por patología basal evoluciona con neumonía necrotizante que no requiere nuevas intervenciones quirúrgicas. Se da de alta el 08/05/2018 y completa tratamiento antibiótico por 28 días.

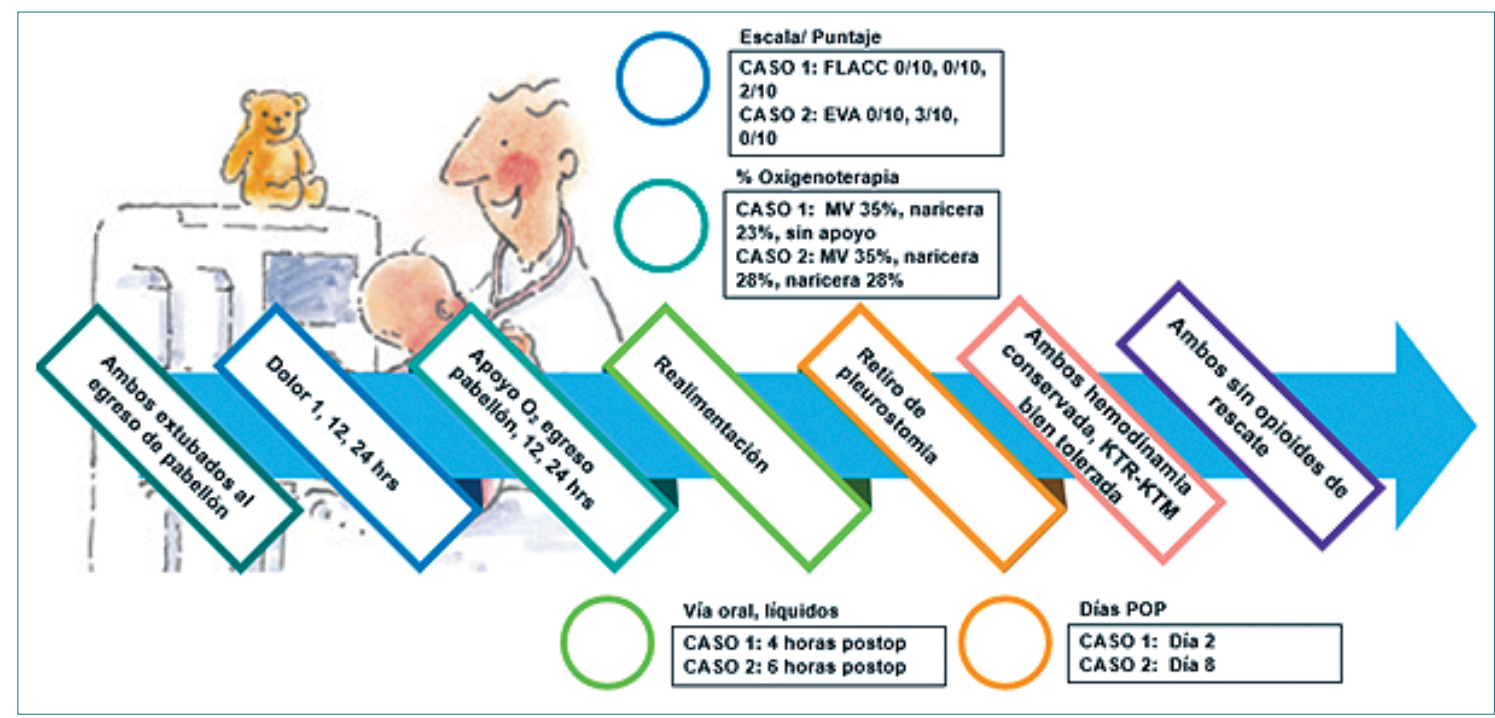

Figura 5. Resumen de resultados. OUTCOME PRIMARIO: (1) Dolor: FLACC - EVA. (2) Requerimiento opioides de rescate. OUTCOME SECUNDARIO: (1) Requerimiento de oxígeno; (2) Tiempo Realimentación; (3) Tolerancia KNT. 


\section{Discusión}

La toracotomía abierta es un procedimiento muy doloroso, cuyas consecuencias pueden entorpecer la evolución del paciente al disminuir la expansión pulmonar en un niño que intenta no tener dolor. Vemos el caso de una lactante cuya alternativa de analgesia epidural no era opción por no tener disponible trócar ni catéter adecuado para su tamaño. Se realizó bloqueo ESP, a diferencia de otros reportes, con punción única y sin instalación de catéter continuo. La paciente no tuvo dolor postoperatorio que entorpeciera su alimentación ni la terapia kinesiológica, siempre toleró el drenaje pleural, tuvo reexpansión torácica completa precoz y logró un egreso hospitalario a las 72 horas (Figura 5).

Por otro lado, tenemos un escolar con cuadro grave de neumonía que evoluciona con dolor pleurítico antes de la resolución quirúrgica. A pesar de que la VATS es un procedimiento menos doloroso que una toracotomía, en este caso requería mantención de drenaje pleural, lo cual en su mayoría es mal tolerado por la población pediátrica, por lo que se decide bloqueo regional ESP, una punción y sin instalación de catéter continuo.

Al igual que la lactante del primer caso clínico, el segundo paciente presenta dolor leve las primeras 12 h y luego se mantiene sin dolor las próximas 48 h, lo que permite realimentación oral a las 4 horas postoperatorias y el retiro del drenaje al septimo día. En su evolución fue fundamental la tolerancia a la kinesioterapia las primeras $48 \mathrm{~h}$.

El bloqueo regional ESP es una alternativa importante a la analgesia peridural en cirugía torácica pediátrica y debería ser considerada de manera más frecuente. La base del control del dolor postoperatorio en la actualidad siempre considera una aproximación multimodal y el bloqueo regional en esta población permitiría la utilización de dosis mínimas de opioides de rescate, eliminando los conocidos efectos secundarios y probablemente la prolongación de la estadía en UCl. Además, se disminuyó la posibilidad de toxicidad por anestésicos locales, más frecuente en esta población y en especial en lactantes.

En los casos expuestos no fue necesario dosis alguna de opioides. Al realizar la técnica de bloqueo ESP en punción única, sin colocación de catéter continuo, además se disminuyó la posibilidad de infección del sitio de punción y la posibilidad de pérdida de catéter por desplazamiento.

\section{Puntos importantes}

- El bloqueo ESP es una nueva opción terapéutica para el manejo de dolor postoperatorio en tórax y sería útil y segura para la población pediátrica, incluyendo lactantes menores.

- Permite menor utilización de opioides de rescate. En los casos expuestos no fue necesario dosis alguna de opioides en el postoperatorio. Manejo solo con AINEs y paracetamol.

- Permite evitar las complicaciones de la analgesia peridural.

- Sería suficiente una técnica con punción única, sin instalación de catéter continuo. Esto disminuye la posibilidad de toxicidad por anestésicos locales, la probabilidad de infección en el sitio de punción y el riesgo de pérdida del catéter.

- Ambos pacientes tuvieron una buena analgesia posoperatoria, lograron ciclos de sueño-vigilia normales, se realimentaron de forma precoz y toleraron muy bien la kinesioterapia.

\section{Referencias}

1. Forero M, Rajarathinam M, Adhikary S, Chin KJ. Erector spinae plane (ESP) block in the management of post thoracotomy pain syndrome: A case series .Scand J Pain. 2017 Oct;17(1):3259. https://doi.org/10.1016/j. sjpain.2017.08.013 PMID:28919152

2. Kehlet $\mathrm{H}$, Wilkinson $\mathrm{RC}$, Fischer HB, Camu F; Prospect Working
Group. PROSPECT: evidencebased, procedure-specific postoperative pain management. Best Pract Res Clin Anaesthesiol. 2007 Mar;21(1):149-59. https://doi. org/10.1016/j.bpa.2006.12.001 PMID:17489225

3. Forero $M$, Rajarathinam $M$, Adhikary S, Chin KJ. Continuous Erector Spinae Plane Block for Rescue Analgesia in Thoracotomy After Epidural Failure: A Case Re- port .A A Case Rep. 2017 May;8(10):254-6. Disponible en: http://insights.ovid.com/crossref?20097-201705150-00003 https://doi.org/10.1213/ XAA.0000000000000478 PMID:28252539

4. Forero M, Adhikary SD, Lopez H, Tsui C, Chin KJ, Hons M. The Erector Spinae Plane Block A Novel Analgesic Technique in Thoracic Neuropathic Pain. 2016;41(5):1-7. 
5. Muñoz F, Cubillos J, Bonilla AJ, Chin KJ. Erector spinae plane block for postoperative analgesia in pediatric oncological thoracic surgery. Can J Anesth Can d'anesthésie .2017;64(8):880-2. Disponible en: http://link.springer. com/10.1007/s12630-017-0894-0

6. Seidel R, Gray AT, Wree A, Schulze M. Surgery of the axilla with combined brachial plexus and intercostobrachial nerve block in the subpectoral intercostal plane. Br J Anaesth. 2017 Mar;118(3):472-4. https:// doi.org/10.1093/bja/aex009 PMID:28203727

7. Chin KJ, Adhikary S, Sarwani $\mathrm{N}$, Forero $\mathrm{M}$. The analgesic efficacy of pre-operative bilateral erector spinae plane (ESP) blocks in patients having ventral hernia repair. Anaesthesia. 2017 Apr;72(4):452-60. https:// doi.org/10.1111/anae.13814 PMID:28188621

8. Kumar A, Hulsey A, MartínezWilson H, Kim J, Gadsden J. The Use of Liposomal Bupivacaine in Erector Spinae Plane Block to Minimize Opioid Consumption for Breast Surgery. A A Case Reports. 2017;16(16):1. Disponible en: http://insights.ovid.com/ sref?an=01720097-900000000-

\section{8}

9. Tanaka N, Ueshima H, Otake $\mathrm{H}$. Erector spinae plane block for combined lovectomy and radical mastectomys .J Clin Anesth. 2018 Mar;45:27-8. https://doi.org/10.1016/j.jclinane.2017.12.012 PMID:29274543

10. Ueshima H, Otake H. Clinical experiences of ultrasound-guided erector spinae plane block for thoracic vertebra surgery. J Clin Anesth. 2017;38 (October 2017): 137. Disponible en: https://doi.org/10.1016/j.jclinane.2017.10.021

11. Scimia P, Basso Ricci $E$, Droghetti A, Fusco P. The ultrasoundguided continuous Erector Spinae plane block for postoperative analgesia in video- assisted toracoscopic lobectomy .Reg Anesth Pain Med. 2017 Jul/ Aug;42(4):537. Disponible en: http://insights.ovid.com/crossref?115550-201707000-00021 https://doi.org/10.1097/ AAP.0000000000000616 PMID:28632673

12. Ueshima H, Otake H. Erector spinae plane block provides effective pain management during pneumothorax surgery .J Clin Anesth. 2017 Aug;40(April):74. https://doi.org/10.1016/j.jclina- ne.2017.04.016 PMID:28625453

13. De la Cuadra-Fontaine JC, Concha $\mathrm{M}$, Vuletin $\mathrm{F}$, Arancibia $\mathrm{H}$. Continuous Erector Spinae Plane block for thoracic surgery in a pediatric patient. Pediatr Anesth .2018;28(1):74-5. Disponible en: http://doi.wiley.com/10.1111/ pan.13277 https://doi. org/10.1111/pan.13277.

14. Gaio-Lima C, Costa CC, Moreira JB, Lemos TS, Trindade HL. Bloqueo continuo en el plano del músculo erector del espinal para analgesia en cirugía torácica pediátrica: informe de un caso .Rev Esp Anestesiol Reanim. 2018 May;65(5):287-90. https://doi. org/10.1016/j.redar.2017.11.010 PMID:29370900

15. Chin KJ, Malhas L, Perlas A. The erector spinae plane block provides visceral abdominal analgesia in bariatric surgery: a report of 3 cases. Reg Anesth Pain Med. 2017 May/Jun;42(3):3726. https://doi.org/10.1097/ AAP.0000000000000581 PMID:28272292

16. Ueshima $\mathrm{H}$, Otake $\mathrm{H}$. J Clin Anesth. 2018 Feb;44:41. Clinical experiences of erector spinae plane block for children. Disponible en: https://10.1016/j.jclinane.2017.10.021 